\title{
Performance Improvement of IEEE 802.22 WRAN Physical Layer
}

\author{
Sharad Jain ${ }^{1}$, Raksha Upadhyay ${ }^{2}$ \\ ${ }^{I}$ (Electronics and Communication Engineering, Lord Krishna College of Technology/RGPV, India) \\ ${ }_{2}^{2}$ (Department of Electronics \& Communication Engineering, IET/ DAVV, India)
}

\begin{abstract}
The spectrum available for the wireless services is limited, the increased demand of wireless application has put a lot of limitations on the utilization of available radio spectrum. For the efficient spectrum utilization for wireless application IEEE 802.22 standard i.e. WRAN (Wireless Regional Area Network) is developed which is based on cognitive radio technique that senses the free available spectrum. It allows sharing of geographically unused channels allocated to the TV Broadcast Service, without interference.

In this paper we are evaluating the performance of WRAN over physical layer with QPSK, 16-QAM and 64-QAM modulation with Convolution coding with code rate of 1/2, 2/3, 3/4, 5/6 and obtaining the BER curves for rician channel. Simulation is performed in MATLAB.
\end{abstract}

Keywords - CC, CP, CR, OFDMA, PHY Layer, WRAN

\section{INTRODUCTION}

The TV broadcast bands in the high-VHF/low-UHF range are ideal for covering large areas in sparsely populated rural environments because of lower industrial noise and ionospheric reflections, reasonable antenna sizes, and good non-line-of-sight (NLOS) propagation characteristics.

IEEE 802.22 is standard for wireless regional area network (WRAN) using white spaces the TV frequency spectrum. The development of the IEEE 802.22 WRAN standard is aimed at using cognitive radio (CR) techniques to allow sharing of geographically unused spectrum allocated to the television broadcast service, on a non-interfering basis, to bring broadband and access to hard-to-reach, low population density area, typical of rural environments, and is therefore timely and has the potential for a wide applicability worldwide.

CR effectively reuses the finite frequency resources by intelligent recognition of ideal frequency bands using spectrum sensing [1][2][3]. IEEE 802.22 WRANs are designed to operate in the TV broadcast bands while ensuring that no harmful interference is caused to the incumbent operation (i.e., digital TV and analog TV broadcasting) and low-power licensed devices such as wireless microphones [4].

OFDMA is also a candidate access method for the IEEE 802.22 wireless regional area network. WRAN is fixed point to multi-point (PMP) system and the base station (BS) and the Consumer premise Equipments (CPEs) can be connected with each other in both line-of-sight (LOS) and non line-of-sight (NLOS) situations. The standard typical support range is $30 \mathrm{~km}$. meeting the demands of rural areas, but based on propagation conditions it may cover up to $100 \mathrm{~km}$. The minimum data rate of the system is $1.5 \mathrm{Mb} / \mathrm{s}$ in the downstream direction, i.e. from BS to CPE and $384 \mathrm{~kb} / \mathrm{s}$ in the upstream direction, i.e. from CPE to BS. It is expected that a BS supports up to $255 \mathrm{CPEs}[8]$.

\section{Role Of Physical Layer AND Mac Layer}

The physical layer \& MAC layer performs important task in the operation of WRAN.

The physical layer must be able to adapt to different conditions. Also it should be flexible for switching from one channel to another without error in Transmission and hence this feature of physical layer is known as dynamic frequency hopping required for adjusting the band width dynamically, modulation and coding scheme.

Physical layer is responsible for transmission of Data. MAC layer is based on cognitive Radio technology. The MAC layer has two structures namely frame and super frame. A super frame will be formed by no. of frames. The Super Frame will have a super frame control header and a preamble. These will be sent by the base station in every channel.

\section{PHYSICAL LAYER SPECIFICATION}

The physical layer of IEEE 802.22 is more or less similar to IEEE 802.16e and is based on OFDMA scheme in the Time Division Duplex (TDD) mode. In the future, It is expected to use Frequency Division Duplex mode (FDD).

The mandatory code for Forward Error Correction is Binary Convolution coding with code rate 1/2. Other optional codes are Convolution Turbo code (CTC), Shortened Block Turbo Code (SBTC), and Low Density 
Parity Check Code (LDPC) to increase the performance. To achieve faster data rate in low noise channels, puncturing can be used to achieve code rates of $2 / 3,3 / 4$, and 5/6[5].

There are fourteen modes of transmission, twelve of which are dedicated to data. The digital modulation types used in this standard are QPSK, 16-QAM, and 64-QAM. OFDMA symbols are created using the 2048 FFT on 6,7 , and $8 \mathrm{MHz}$ bandwidths which consist of 1440 data subcarriers, 240 pilot carriers, and 368 guards and DC subcarriers [6]. The cyclic prefix length can be $1 / 4,1 / 8,1 / 16$, and 1/32 of OFDMA symbol duration.

\section{IEEE 802.22 PHY LAYER SYSTEM MODEL OVERVIEW}

It supports multiple physical specifications. The 802.22 OFDMA PHY is based on OFDMA modulation, which includes OFDM modulation \& subcarrier allocation.

\section{OFDMA}

OFDMA is the multi user version of OFDM. OFDM belongs to a special transmission scheme of multicarrier modulation in which high bit rate data stream is divided into several parallel lower bit rate streams. Each stream is modulated on separate carrier called, subcarriers. OFDM is the most spectral efficient version of the multicarrier modulation. In OFDM all the subcarrier are orthogonal to each other over one symbol duration. Because of the orthogonality there is no need of non overlapping subcarriers to eliminate the ICI [7] now the subcarriers overlap to each other thus saves the bandwidth. The no. of subcarriers in the OFDMA symbol is always the size of FFT. There are no. of subcarriers in the OFDMA symbol as follows

- Data carriers- carries the useful data

- Null carriers-no data is transmitted on these carriers \& are used to prevent leakage of energy into adjacent channels

- Pilot carrier -are used for channel estimation \& synchronization purpose.

In OFDMA to enable the multiple access sub channels are used which are formed by grouping the data subcarriers. The all subcarrier that are used to make a subchannel are distributed across all the subcarriers. Number of different subchannels are allocated to the particular user in order to send \& receive data. There are two parts in one OFDMA symbol in time domain. The cyclic prefix (CP) time \& useful symbol time. CP is used to remove the effect of ISI \& ICI in multipath scenario.

CP is the copy of the last part of the OFDM symbol which is appended in the front of the transmitted OFDM symbol. The length of CP or guard time interval must be chosen such that it is longer than the maximum delay spread of the multipath channel[7]. The figure 1 shows the advantage of $\mathrm{CP}$.

\begin{tabular}{|c|c|}
\hline Cyclic prefix & OFDM symbols \\
\hline$\longleftarrow N_{s}$ samples $\longrightarrow$ & N samples $\longrightarrow$
\end{tabular}

Fig. 1- Cyclic Prefix addition

\section{Simulation Model}

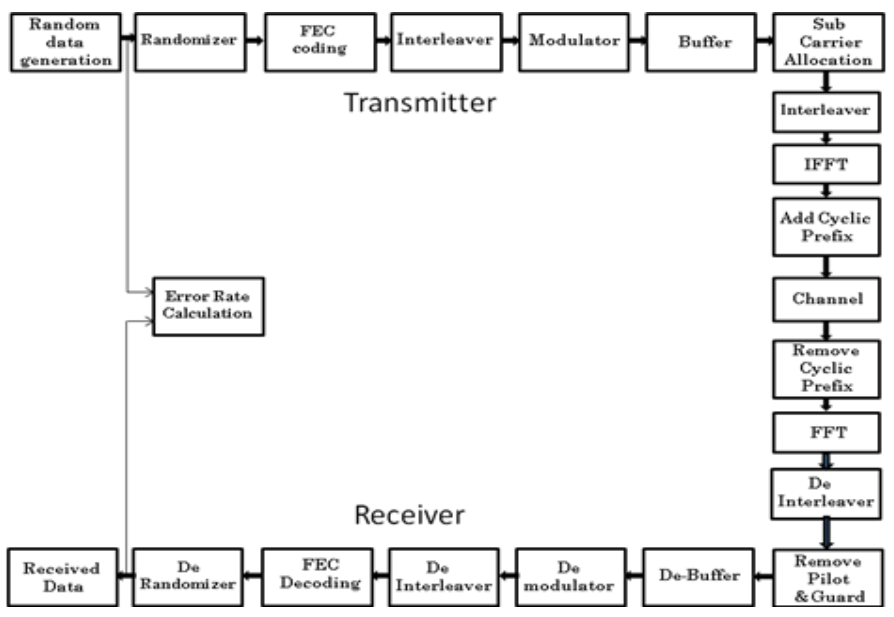

Fig.2-Simulation setup for PHY layer

\subsection{Random Data Generation}

Random binary data is generated here for simulation purpose. 
2.2 Randomizer

Convert the binary sequence to the random sequence to avoid long sequence of ' 0 ' \& ' 1 ' to improve Synchronization.

2.3 FEC coding

Convolution code with code rate $1 / 2,2 / 3,3 / 4$ and $5 / 6$ is used as FEC.

2.4 Interleaver

It rearranges the ordering of the sequence of the symbols in predefined manner. Two types of interleaving are used i.e. Time Interleaving \& Frequency interleaving.

2.5 Modulator

QPSK, 16-QAM, and 64-QAM modulations are used for simulation.

2.6 Buffer

Buffer is used to stack the data.

2.7 Subcarrier allocation

Pilots and guard subcarrier are inserted in this stage.

2.8 IFFT

Inverse fast Fourier Transform converts the frequency domain data set into corresponding time domain. It also generates the sample of the waveform which satisfies the condition of orthogonality.

2.9 Cyclic prefi

$\mathrm{CP}$ copies the last section of a symbol \& paste it to the beginning of symbol. After these operations the data are delivered to the channel[8].

2.10 Channel

RICIAN channel is used for simulation in this paper.

\section{Forward error correction}

Convolution code with code rate $1 / 2$ is the only mandatory code for WRAN system. They operate on data stream, not static block.

Convolutional codes have memory that uses previous bits to encode or decode following bit. It is denoted by $(\mathrm{n}, \mathrm{k}, \mathrm{L})$ where $\mathrm{L}$ is the memory depth. In Matlab Trellis description is used to define encoder connections. Viterbi algorithm is used for decoding.

\section{Simulation Parameter}

Total no. of subcarriers: 2048

No. of guard subcarriers: 368

No. of used subcarrier: 1680

No. of data subcarriers: 1440

No. of pilot subcarriers: 240

Guard time to useful symbol time ratio: $1 / 8$

\section{Results}

As shown in Fig 3, the uncoded BER curves obtained by simulation. Since the FEC blocks are not applied to the data in this case, differences in code rates have no effect on results. In order to investigate the effect of FEC blocks on the system performance, convolutional coding is used.

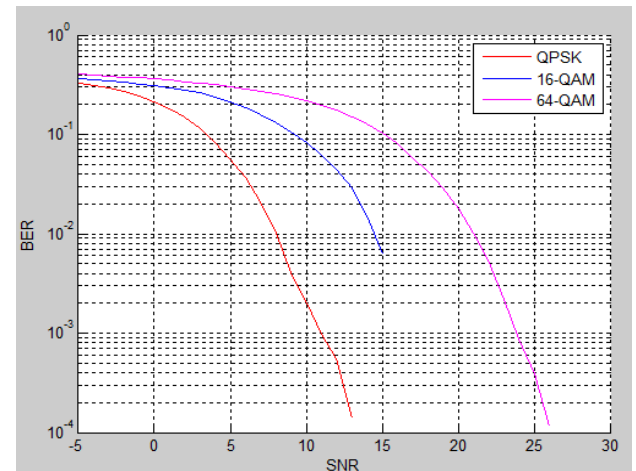

Fig.3- Uncoded BER for QPSK, 16-QAM, and 64-QAM

Figs. 4, 5, and 6 show simulation results of the system coded BER versus SNR with different code rates for QPSK, 16-QAM, and 64-QAM respectively. As expected, the BER of higher modulation schemes with high data rates are more sensitive to SNR degradation. e.g. to achieve a BER equal to $10^{-2}$ in QPSK and $1 / 2$ code rate, 
an SNR above 6.6dB is needed. .For 16-QAM and 3/4 code rate, an SNR above $16 \mathrm{~dB}$ is needed. However for $64-\mathrm{QAM}$ with $5 / 6$ code rate the minimum required SNR is $22.5 \mathrm{~dB}$.

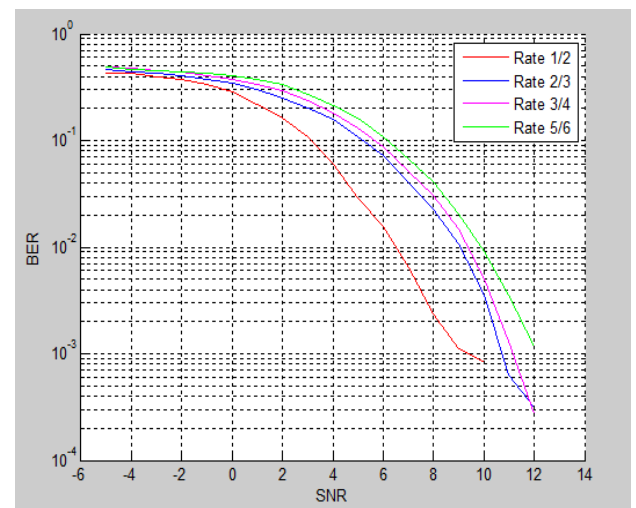

Fig.4- Coded BER for QPSK with different code rates

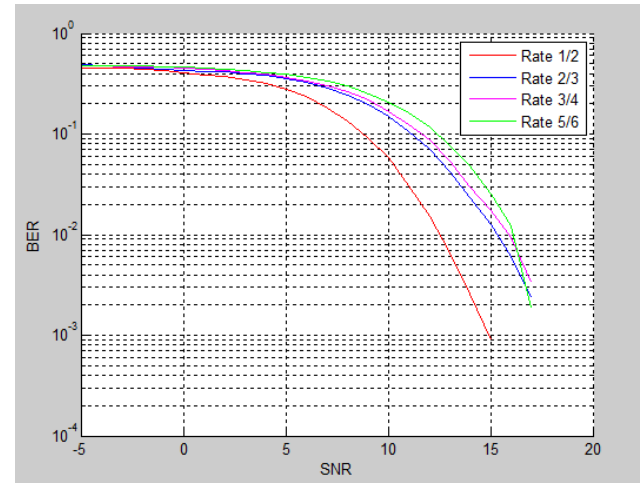

Fig.5- Coded BER for 16-QAM with different code rates

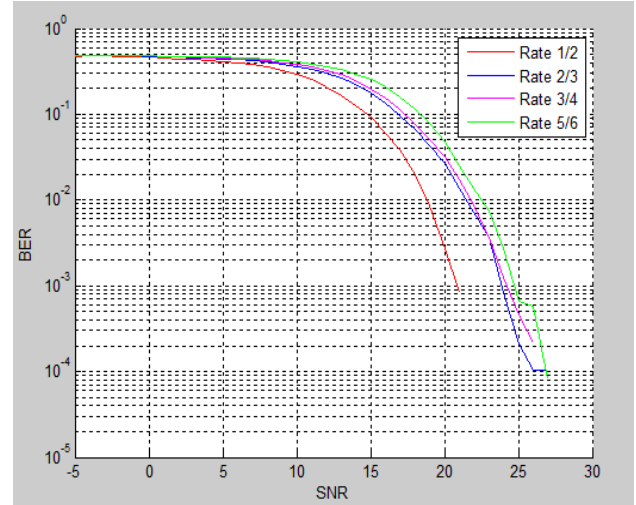

Fig.6 -Coded BER for 64-QAM with different code rates

\section{Conclusion}

In this paper the PHY layer of IEEE 802.22 has been modeled in MATLAB. The BER curves for different modulation schemes and code rates in rician channel are obtained by simulation According to BER curves, It has concluded that for all modulation technique $\mathrm{CC}$ with code rate $1 / 2$ gives significant performance improvement to without coding and other higher code rate.

\section{REFERENCES}

[1] S.Haykin,"Cognitive radio: Brain empowered wireless communications"IEEE J. sel. Areas Comm.Vol.23, no.2, pp.201-220, Feb.2005.

[2] J. Mitola,"Cognitive Radios: Making Software Radios more personal” IEEE Personal Communications, vol.6, no. 4, August1999

[3] J. Mitola,"Cognitive Radios: An integrated agent architecture for software defined radio", PhD Dissersation, Royal inst. Techno.(KTH),Stockholm,Sweden2000.

[4] Functional Requirements for the IEEE 802.22 WRAN standard, IEEE 802.22-05/0007r46, Sep. 
[5] C. Stevenson, G. Chouinard, L. Zhongding, H. Wendong, S. Shellhammer, and W. Caldwell, "IEEE 802.22: The First Cognitive Radio Wireless Regional Area Network Standard", IEEE Mag. Communications, vol 47, Jan. 2009, pp. 130-138, doi:10.1109/MCOM.2009.4752688.

[6] S. H. Hwang, J. S. Um, M. S. Song, C. J. Kim, H. R. Park, and Y. H Kim, "Design and Verification of IEEE 802.22 WRAN

Physical Layer (Invited Paper)," Proc. International Conf. Cognitive Radio Oriented Wireless Networks and Communications (Crown Com 08), IEEE Press, May. 2008, pp. 1-6,doi:10.1109/CROWNCOM. 2008.4562542

[7] Richard van Nee, Ramjee Prasad "OFDM for Wireless Multimedia Communication" Artech house publishers, 2000.

[8] Ahmadi, M., Rohani, E., Naeeni, P.M., \& Fakhraie, S.M.”Modelling \& Performance Evaluation of IEEE 802.22 Physical layer"2nd International Conference on future Computer and Communication- (2010).

[9] J. G. Proakis, Digital Communications, 3rd ed., vol. 2. McGraw-Hill: New York, 1995. 